\title{
An Order-N Study of Dislocations in Homopolar Semiconductors
}

\author{
R.W. Nunes \\ Departamento de Fúsica, Universidade Federal de Minas Gerais \\ Belo Horizonte, Minas Gerais 30123-970, Brazil \\ Received February, 1998
}

\begin{abstract}
This article surveys the main results of the author's work, in collaboration with David Vanderbilt and John Bennetto, on the application of the order- $\mathrm{N}$ density-matrix approach, together with $a b$ initio methods, to investigate the atomic structure of dislocation cores in the homopolar semiconductors silicon, carbon, and germanium. In these systems, the predominant dislocations are the $30^{\circ}$ and the $90^{\circ}$ partial dislocations. For the three materials, the nature of the reconstruction at the core of the $90^{\circ}$-partial dislocation is considered. Both the traditional single-period and our recently proposed double-period core structures are investigated. The double-period geometry is found to be the ground-state structure in all three cases. For silicon, we have also investigated in detail the structure and dynamics of point excitations (kinks, solitons, and kink-soliton complexes) in the cores of the $30^{\circ}$ partial dislocation and the single-period geometry of the $90^{\circ}$ partial. Our calculated formation energies and migration barriers for these excitations are in good agreement with available experimental results. Furthermore, we have examined the reactions by which high-energy kinks relax into low-energy ones by soliton emission.
\end{abstract}

\section{Introduction}

The fundamental understanding of plasticity in solids requires a knowledge of the atomistic structure at the cores of dislocations. Dislocation motion occurs by the propagation of point excitations (kinks) that occur within the dislocation core. The multiplicity and structure of these point excitations are connected with the underlying lattice symmetries and the nature of the reconstruction in the core.[1] Recent advances in computer power and computational methodology have led to an active area of research focused on the theoretical study of the atomistic structure of these dislocation cores and their defects.[2-14]

A wealth of experimental information is available about the properties of dislocations in semiconductors.[15-21] In tetrahedrally bonded systems, such as silicon ( $\mathrm{Si}$ ), the two most frequently occurring dislocations are the $30^{\circ}$ and the $90^{\circ}$ partial dislocations, lying on $\{111\}$ planes along [110] directions.[15-17] Both are known to dissociate into pairs of partial dislocations bounding a ribbon of intrinsic stacking fault.[15-17] Dissociation lowers the strain energy and is made energetically favorable by the low energy of the stacking fault in these materials. The resulting $90^{\circ}$ and $30^{\circ}$ partials are believed to undergo core reconstruction, which eliminates the unsaturated bonds, thus restoring the fourfold coordination of the atoms at the cores. This picture is consistent with the low density of dangling bonds, as suggested by EPR measurements.[15,16]

Given their technological importance, the detailed understanding of the atomic structure at the dislocation cores in these materials is of great interest, since dislocations influence both the electronic and the mechanical properties of semiconductor devices. In the particular case of $\mathrm{Si}$, the theoretical study of the dislocation cores at the atomistic scale has revealed a rich structure of point excitations (kinks and reconstruction defects) in the core of the $30^{\circ}$ and $90^{\circ}$ partials. $[2-4,6,8,13]$

Here, I survey the results of our investigation of the atomic structure of dislocation cores in homopolar semiconductors. This study was carried out using an order- $\mathrm{N}$ density-matrix technique, [22] in the context of a tight-binding Hamiltonian for a quantum-mechanical description of the electronic structure, as discussed in the next section.[23] In Sec. III, the core reconstruction of the $90^{\circ}$ partial in Si, carbon (C), and germanium (Ge) is reviewed. Our calculations indicated a new ground-state structure for the defect, a feature which we also investigated by means of $a b$ initio calculations. For $\mathrm{Si}$, the nature of the core reconstruction of the $30^{\circ}$ partial is also discussed (Sec. V), as well as the structure and dynamics of the point excitations (kinks and 
reconstruction defects, the latter being commonly referred to as solitons) occurring in the cores of the two partials (Secs. IV and V). A comparison with available experimental results for kink formation energies and migration barriers, and some concluding remarks are given in Secs. VI and VII, respectively.

\section{The order-N density-matrix technique}

From a computational point of view, the atomic-scale study of the structure of extended defects in solids, such as dislocations, grain boundaries, and stacking faults, with a proper quantum-mechanical treatment of the electronic structure, is limited by the highcomputational cost associated with the "traditional" approaches. This is also true for problems such as lattice dynamics in amorphous semiconductors, where molecular-dynamics simulations are employed. By traditional, we mean methods in which the electronic Hamiltonian is diagonalized, enabling the calculation of the electronic-structure contributions to the energy and the forces acting on the system. This bottleneck applies to both $a b$ initio and tight-binding approaches, whenever diagonalization is used, since the computational time scales with the third power of the number of atoms in the system $\left[\mathcal{O}\left(N_{a t}^{3}\right)\right]$. In these approaches, once the eigenvalue equation $H\left|\psi_{n}\right\rangle=\varepsilon_{n}\left|\psi_{n}\right\rangle$ is solved, the electronic total energy and the $\alpha$-th component of the electronic contribution to the force acting on the $i$-th atom are given, respectively, by $E_{\text {tot }}=\sum_{n} \varepsilon_{n}$ and $F_{\alpha}^{i}=\sum_{n}\left\langle\psi_{n}\left|\partial H / \partial r_{\alpha}^{i}\right| \psi_{n}\right\rangle$ (the sums on the index $n$, appearing in these and the following equations, run over the occupied electronic states).

In recent years, several approaches aiming at solving the electronic-structure problem in a linear-scaling or $\mathcal{O}\left(N_{a t}\right)$ fashion have been proposed. In our study, we use the density-matrix method introduced by $\mathrm{Li}$, Nunes, and Vanderbilt (LNV), [22] in which the problem is formulated in terms of the one-particle densitymatrix, $\rho=\sum_{n}\left|\psi_{n}\right\rangle\left\langle\psi_{n}\right|$, associated with the electronic Hamiltonian. In terms of $\rho$, integrated quantities such as $E_{\text {tot }}$ and $F_{\alpha}^{i}$ are written simply as $E_{\text {tot }}=\operatorname{tr}[\rho H]$ and $F_{\alpha}^{i}=\operatorname{tr}\left[\rho \frac{\partial H}{\partial r_{\alpha}^{i}}\right]$, where $\operatorname{tr}[A]$ is the trace of the matrix $A$. In this formulation, the diagonalization of the Hamiltonian is avoided, but one is now left with the need to enforce the idempotency of the density matrix, i.e. the condition $\rho=\rho^{2}$, which ensures the proper fermionic filling of the electronic levels. In terms of the eigenvalues $\lambda_{\rho}$ of $\rho$, idempotency implies that $\lambda_{\rho}=1$ for energy levels below the chemical potential (or fermi level) $\mu$, and $\lambda_{\rho}=0$ for levels above $\mu$.
In the LNV method, the idempotency requirement is enforced indirectly, by introducing the purification transformation

$$
\tilde{\rho}=3 \rho^{2}-2 \rho^{3} \text {. }
$$

In essence, $\rho$ is now treated as a trial density matrix, while $\tilde{\rho}$ is the physical density matrix, in the sense that the required observables are now written as $\langle O\rangle=\operatorname{tr}[\tilde{\rho} \hat{O}]$, where $\hat{O}$ is the operator associated with observable $O$. The ground-state $\tilde{\rho}$ is found by minimizing the electronic total energy

$$
E_{t o t}=\operatorname{tr}[\tilde{\rho} H]=\operatorname{tr}\left[\left(3 \rho^{2}-2 \rho^{3}\right) H\right]
$$

with respect to the matrix-elements of $\rho$, in the chosen tight-binding basis. The transformation given by Eq. (1) is such that, upon minimization of Eq. (2), the occupation of the eigenvalues below (above) $\mu$ is driven to its physical value of $1(0)$, as desired. The solution is stable, since Eq. (1) has stable fixed points at $\lambda_{\rho}=0,1$. Moreover, the LNV solution takes advantage of the fact that $\rho$ is exponentially localized in real-space, i.e., for $\left|\vec{R}_{j}-\vec{R}_{i}\right| \rightarrow \infty, \rho_{i j} \propto \exp \left(-\left|\vec{R}_{j}-\vec{R}_{i}\right|\right)$, where $i$ and $j$ are tight-binding orbitals centered at sites $R_{i}$ and $R_{j}$, respectively. Linear-scaling is then achieved by truncating the density matrix beyond a chosen cutoff radius $R_{c}$. The method is variational with respect to both $\rho$ and $R_{c}$, and becomes exact in the limit $R_{c} \rightarrow \infty$. This ensures that the forces can be obtained by a HellmanFeyman expression

$$
F_{\alpha}^{i}=\operatorname{tr}\left[\left(3 \rho^{2}-2 \rho^{3}\right) \frac{\partial H}{\partial r_{\alpha}^{i}}\right] .
$$

\section{Core reconstruction of the $90^{\circ}$ partial dislocation}

To date, the majority of the theoretical works on the atomic-structure of dislocations in semiconductors have addressed the structure of the $90^{\circ}$ partial dislocation. From these studies, a consensus had emerged at the theoretical level, about the nature of the reconstruction at the core of the defect. In its unreconstructed configuration, the core of the $90^{\circ}$ partial displays a zigzag chain of threefold-coordinated atoms running along the dislocation direction, with broken bonds lying nearly parallel to the slip plane. Mirror symmetry planes, along the dislocation direction, are present is this configuration. A reconstruction that breaks the mirror symmetry of the unreconstructed core, while preserving the lattice periodicity along the line, is shown in Fig. 1(a). In the following discussion, this symmetry-breaking reconstruction is referred to as the single-period (SP) reconstruction. In all our figures the shaded area represents 
the stacking fault, and the dislocation line is indicated by the boundary between shaded and unshaded areas. White (black) atoms lay above (below) the glide plane; besides the bonds in the plane shown in the figures, each atom is bonded to another either above or below it. Notice that all dangling bonds have been eliminated, and all the atoms are fourfold coordinated. This reconstruction was predicted to be substantially lower in energy than both the unreconstructed and the related quasifivefold-reconstructed cores (in the latter, the core is allowed to relax, with the constraint that the mirror symmetry is preserved), $[2,7,9-11]$ hence being the one expected to occur in nature.
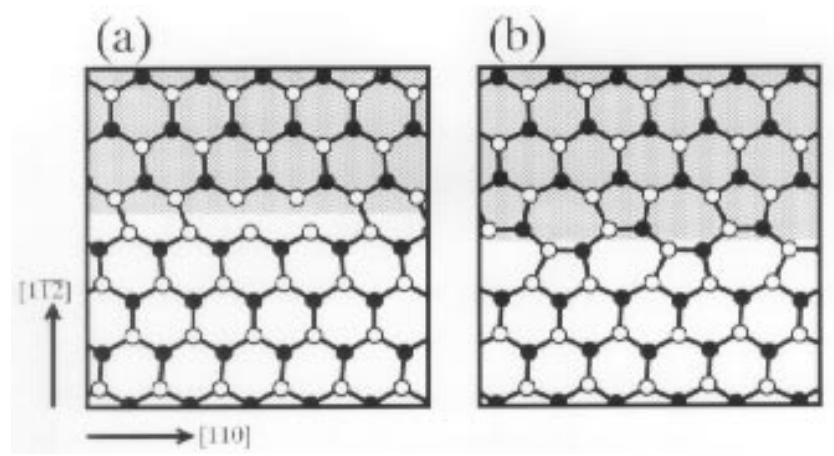

Figure 1. (a) The single-period (SP) symmetry-breaking core reconstruction of the $90^{\circ}$ partial dislocation in homopolar semiconductors, viewed from above the (111) slip plane. Black (white) atoms lie below (above) the slip plane and the shaded area indicates stacking fault. Crystalline directions are also shown. (b) The double-period (DP) symmetrybreaking reconstruction.

However, our recent theoretical work, on the core reconstruction of this dislocation in $\mathrm{Si}$, has indicated otherwise. In Ref.[3], we proposed an alternative core structure for the Si $90^{\circ}$ partial in which, in addition to symmetry breaking of the SP core, the periodicity along the dislocation line is doubled. This doubleperiod (DP) reconstruction, shown in Fig. 1(b), can be derived from the SP one by introducing alternating kinks at every lattice site along the core. Its geometry is consistent with all available experimental information about the $90^{\circ}$ partial. Being fully reconstructed, the DP structure does not give rise to deep-gap states which would show an EPR signal. (EPR measurements in Si indicate a rather small density of dangling bonds in the core of dislocation.[15-17] Surely, this is also a feature of the SP core. Moreover, both cores consist entirely of fivefold, sixfold, and sevenfold rings, both being consistent with images produced by transmission electron microscopy, at the current level of resolution of this technique.[21] While the experiments appear unable to decide clearly on the issue, our theoretical results in Ref. [3] show the DP structure to be lower in energy than the SP one, by means of Keating-potential, total-energy tight-binding (TETB), and ab initio localdensity (LDA) calculations.

In Ref. [4], we extended the investigation of the DP$\times \mathrm{SP}$-core issue to carbon $(\mathrm{C})$ and germanium $(\mathrm{Ge})$. In that work, we found the DP core to be the groundstate in all three materials. The results for the energetics of the SP and DP cores in $\mathrm{Si}, \mathrm{C}$, and Ge, are given in Table I. The LDA and TETB calculations are in quite good agreement, except in the case of $\mathrm{C}$, where the TETB results appear to underestimate the energy difference between the two structures. To investigate the influence of dislocation interactions on our results we performed TETB calculations for larger cells, as also shown in Table I. Further, we used a Keatingpotential analyses to elucidate the difference between the local-strain fields generated by the two reconstructions. The indication is that the DP core is able to "pack" the atoms more efficiently, as indicated by the smaller average bond-length deviations, at the expense of larger bond-angle deviations. The balance between bond-bending and bond-stretching forces leads to the preference of these materials for the DP core. The Keating-potential results indicate a rather subtle difference between the two structures. While the Keating energy for the Keating-potential relaxed structures indicate the SP core to be favorable in $\mathrm{C}$, the same potential when applied to the LDA-relaxed geometries reproduces the LDA trends for the relative energies, with the DP core being more strongly favored in C.

One last comment is in order about our results on these two core structures. Recently, Lehto and Oberg have claimed that the relative stability between the SP and DP structures could depend on the choice of supercell boundary conditions. In order to use periodic boundary conditions, the net Burgers vector in the supercell must be zero. Hence, the cell must contain at least a dislocation dipole. Bigger et al. [7] have suggested the use of the quadrupole cell used in our work, which is designed in order to avoid the presence of spurious strains associated with a tilt grain boundary that forms in the dipole-cell configuration. (Such boundarycondition effects should disappear in the limit of sufficiently large cells.) In order to elucidate this issue, we have performed fully converged [25] (with respect to supercell size) calculations that show that at least in $\mathrm{Si}$ and $\mathrm{C}$, the DP core is favored, for both the dipole and quadrupole cells, showing that our findings hold regardless of the nature of the boundary conditions. 


\section{IV $90^{\circ}$ partial: solitons and kinks}

Symmetry breaking in the SP core gives rise to a solitonic defect at the boundary between two stretches of the dislocation, in which the direction of the bonds is switched, as shown in Fig. 2. Note the presence of a dangling bond in the core of the defect, which explains its formation energy of $1.45 \mathrm{eV}$. Our result is in reasonable agreement with the $1.2 \mathrm{eV}$ value obtained in the cluster calculations of Ref. [13]. We also computed an energy barrier of only $0.04 \mathrm{eV}$ for soliton propagation along the dislocation line. Such a small barrier shows the soliton to be extremely mobile even at low temperatures. As a test, we performed a molecular dynamics simulation on a supercell containing a soliton and an anti-soliton, initially separated by $9.6 \AA$, on an otherwise defect-free partial dislocation. Remarkably, at a temperature of only $50 \mathrm{~K}$, recombination of the pair took place after only $1.3 \mathrm{ps}$.

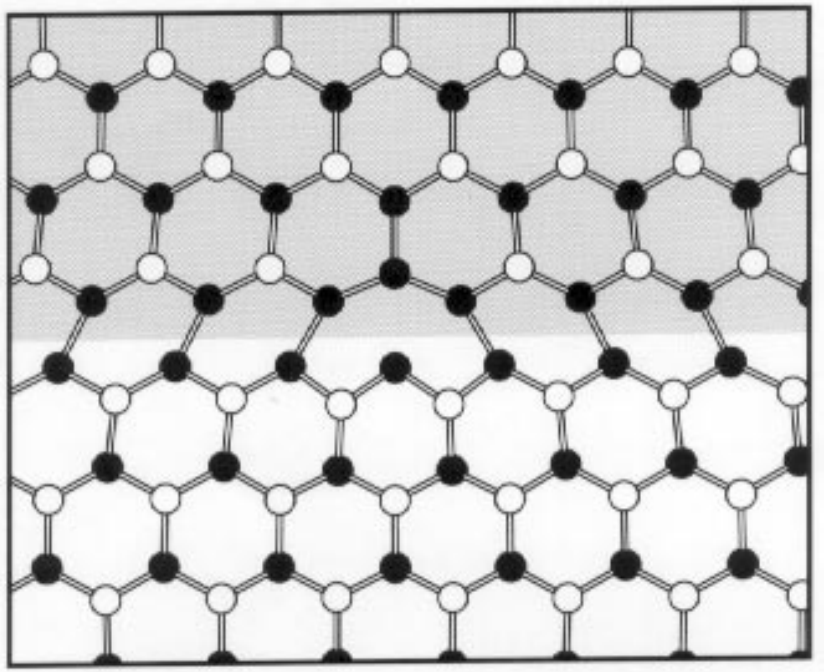

Figure 2. Reconstruction defect (also termed a soliton) in the SP core of the $90^{\circ}$ partial dislocation. The direction of the reconstruction along the dislocation line changes from right to left, going through the defect.

For the study of the kinks in this dislocation core, we adopt a notation based on the sense of the reconstruction, going into and out of the kink, as shown in Fig. 3. Thus, ' $L$ ' and ' $R$ ' denote the direction of the core reconstruction on either side of the kink. Referring to Fig. 3(a), the reconstruction will be said to tilt to the 'left' and to the 'right' on the left and right sides of the kink, respectively. Hence, we call this a left-right (LR) kink, the notation following accordingly for the other defects. We actually compute the sum of the energies of the LR and RL kinks shown in Fig. 3(a) and (b), finding a result of $0.24 \mathrm{eV}$ only. The RL and LR kinks are structurally quite similar; they would be related by a two-fold rotation axis normal to the plane of Fig. 3, if it were not for the fact that a stacking fault exists on one side but not the other. Thus we expect the energies of the two kinks to be similar, and assign the average energy of $0.12 \mathrm{eV}$ to each. This result is in good agreement with the first-principle cluster calculations in Ref. [14] (0.1eV), and in perfect agreement with the Tersoff potential values obtained in Ref. [13] (0.12 $\mathrm{eV}$ ). The rather low formation energy can be seen as another indication of the DP core structure, since even individual kinks add little strain over that imposed by the SP core itself. In the formation of the DP core, this additional strain is more than compensated for by the attraction between the LR and RL kinks. We also computed an energy barrier of $1.62 \mathrm{eV}$ for the motion of the LR and RL kinks. Such large energy barriers are associated with the existence of malcoordinated atoms and severe bond distortions at the core of the kink.

There are two additional kink-type defects associated with the $\mathrm{SP}$ reconstruction of the core. These are the RR and the LL defects, shown in Figs. 3(c) and (d). These defects can actually be seen as complexes of a LR or a RL kink together with a soliton. Two LL complexes are possible (only one is shown in Fig. 3), and they share the same "quasi-symmetry" that the LR and RL kinks do, differing only by the position of the fivefold and dangling-bond-containing rings with respect to the stacking fault. These complexes appear to be either unstable or marginally stable against the emission of a soliton, as discussed in Ref. [2]. The dissociation barrier, if present, is basically the soliton migration barrier, which indicates that these complexes should dissociate very easily at moderate temperatures. This was confirmed by a simulation performed at $300 \mathrm{~K}$, with a supercell containing a pair of RR complexes in each dislocation, separated by a distance of $34.6 \AA$. On the time scale of $1 \mathrm{ps}$, this system undergoes the following relaxation process:

$$
R R+R R \rightarrow(R L+S)+R R \rightarrow R L+(S+R R) \rightarrow R L+L R .
$$


In the first step of this reaction, one of the kink complexes undergoes a soliton-emission reaction, after which the soliton $(S)$ propagates rather easily towards the other RR complex (second step), where it is absorbed by the other RR kink, in the final step. Overall, a dislocation containing a pair of RR complexes relaxes into one containing alternating RL and LR kinks, by means of soliton emission (absorption).
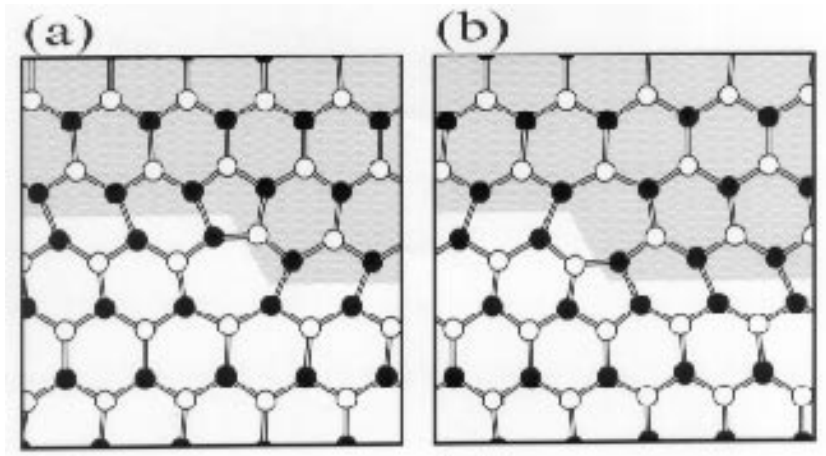

(c)

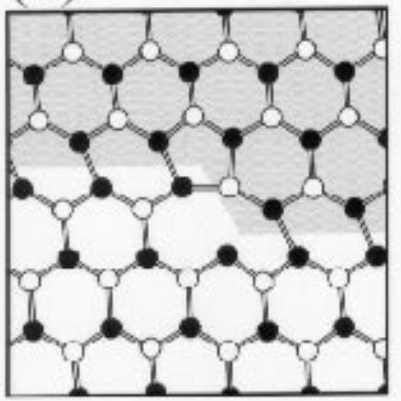

(d)

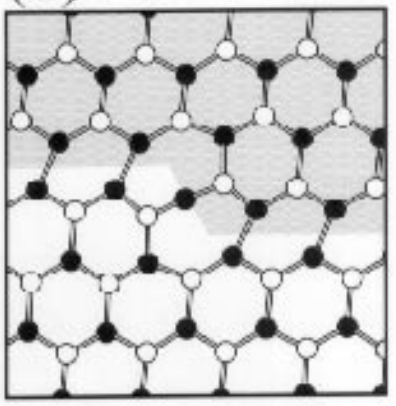

Figure 3. Core structure of kinks and kink-soliton complexes in the SP core. See text for notation. (a) LR kink. (b) RL kink. (c) LL complex $=\mathrm{LR}+$ soliton. (d) RR complex $=$ $\mathrm{LR}(\mathrm{RL})+$ soliton.

\section{V $30^{\circ}$ partial: core reconstruc- tion, solitons and kinks}

In Fig. 4(a), a top view of the atomic structure of the unreconstructed $30^{\circ}$ partial in the glide plane is shown. Notice that the atoms at the core of the defect are threefold coordinated, with a dangling bond lying nearly parallel to the dislocation line. Fig. 4(b) shows the reconstructed structure, where the core atoms bond in pairs along the line, thus restoring their fourfold coordination. This leads to a doubling of the period in the dislocation direction. This reconstruction is well accepted as being the ground-state of the $30^{\circ}$ partial, and has been discussed theoretically by other authors.[6,9,13,17] In Ref [6], it was found to be $0.21 \mathrm{eV} / \AA$ lower in energy than the unreconstructed structure, using a StillingerWeber potential. We find a higher value of $0.36 \mathrm{eV} / \AA$ for the reconstruction energy. The distribution of bond lengths for this structure shows that the reconstruction is indeed strong, with maximum bond-length deviations of only $3.0 \%$ (maximum and minimum bond lengths are $2.42 \AA$ and $2.31 \AA$, respectively) with respect to $\mathrm{Si}$ bulk values $(2.35 \AA)$. To a large extent, the core energy is due to the strain associated with bond-angle distortions at the core of the defect, with bond angles ranging between $\sim 90^{\circ}$ and $\sim 126^{\circ}$ (109.5 is the bulk value). No mid-gap levels are expected for this structure, in accordance with the EPR evidence.[15-17]
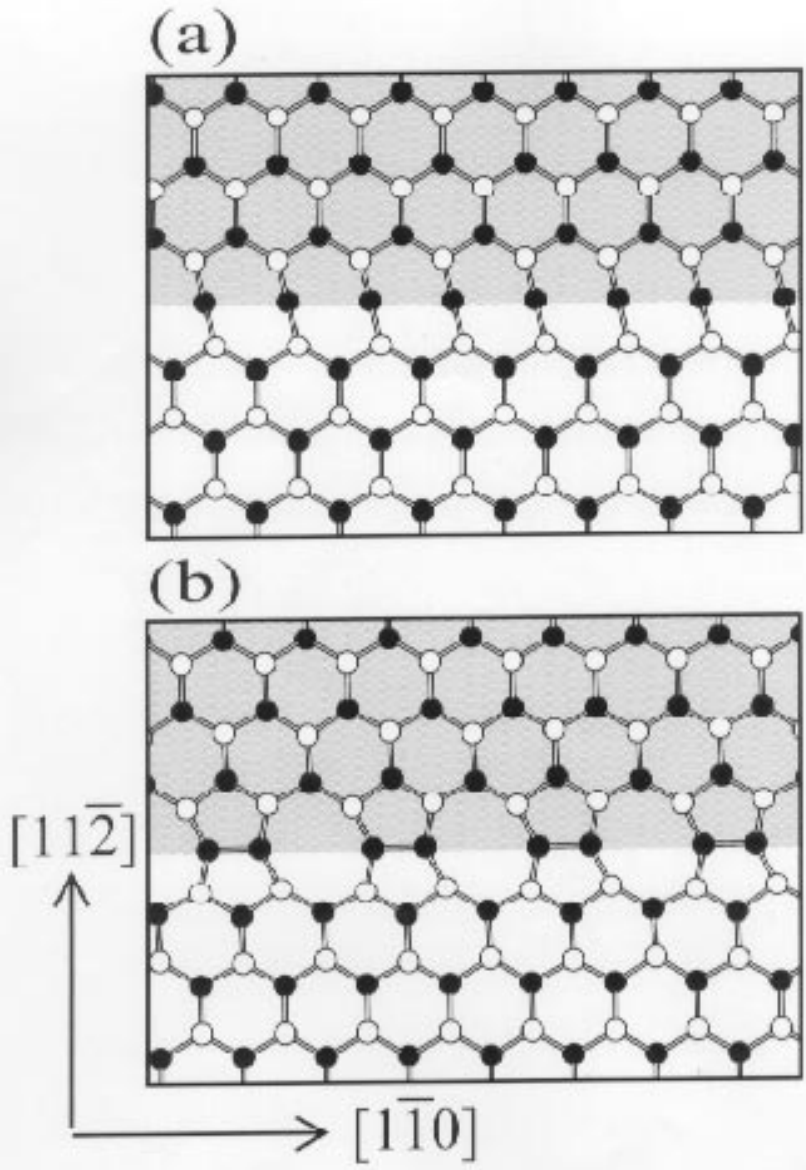

Figure 4. (a) Unreconstructed core of the $30^{\circ}$ partial dislocation in Si. (b) Double-period reconstruction of the same dislocation.

Several core defects are associated with this reconstruction. Like in the case of the $90^{\circ}$ partial, the basic types are kinks and solitons, which also form kinksoliton complexes. A detailed study of these defects is found in Ref. [6], including structural features and energetics under a Stillinger-Weber potential. Our study of this specific dislocation relied on this previous study, adding to it the benefits of a quantum-mechanical treatment of the electronic structure. The formation energies and migration barriers of these excitations are included in Table II. (For the kink-soliton complexes the binding energy is shown in the second column, instead.) 
Fig. 5(a) shows the soliton associated with the core of the $30^{\circ}$ partial. Our value of $1.32 \mathrm{eV}$ for the soliton formation energy is somewhat higher than the value of $0.81 \mathrm{eV}$ obtained in Ref. [6] We believe our result to be more reliable, given the quantum-mechanical nature of our approach, in particular for a defect containing a dangling bond. We also computed the migration barrier for the propagation of the soliton along the dislocation core, obtaining a value of $0.3 \mathrm{eV}$. This indicates that solitons are less mobile in this dislocation than in the $90^{\circ}$ partial. This feature is perhaps reflected on the stability of the kink-soliton complexes in the $30^{\circ}$ partial, which is discussed below. This in contrast with the case of $90^{\circ}$ partial, where complexes are unstable.

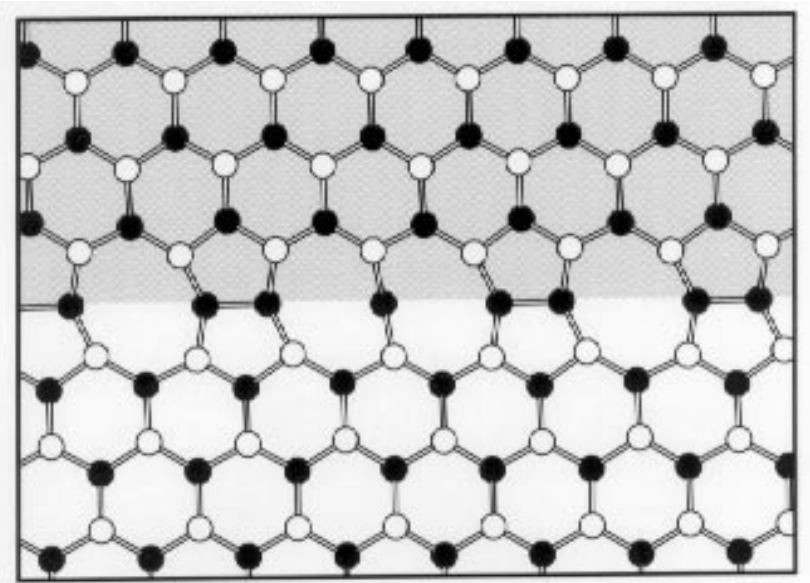

Figure 5. Soliton in the core of $30^{\circ}$ partial dislocation. The phase of the reconstructed bond along the dislocation line is switched, going through the defect.

The period doubling of the reconstructed core gives rise to a multiplicity of kinks in this system. Two distinct families of such defects appear, depending on whether the dislocation "kinks" to the left [Figs. 6(a) and $6(\mathrm{~b})]$ or to the right [Figs. 6(c) and 6(d)]. The period doubling of the core introduces a choice of phase of the core reconstruction both ahead of, and behind, the kink. Of the four configurations generated in this way, two of them (those necessarily containing a coordination defect) are classified as kink-soliton complexes. The remaining two configurations, each with two possible states due to the period doubling, will be classified as "pure" kinks. Thus, in Fig. 6(a-b) we show the left kinks LK and LK', while the right kinks, RK and RK', are shown in Fig. 6(c-d). The energies for each type of kink were computed using the TBTE Hamiltonian, as well as with a classical Keating model [26] in the parameterization suggested in Ref. [27]. In our discussion, we use the Keating model as a tool to examine the local-strain contributions to the energy of each defect. In order to work with more reliable geometries, we analyze the Keating energies for the TB-relaxed structures. (a)

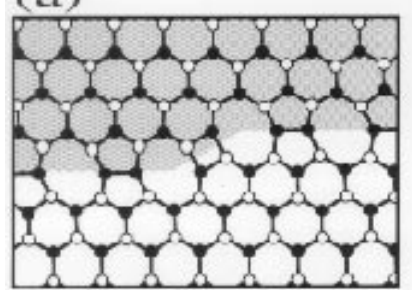

(c)

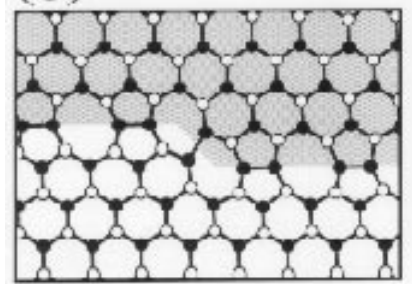

(b)

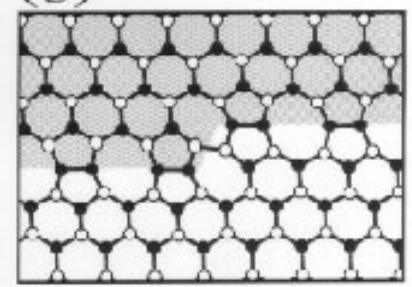

(c)

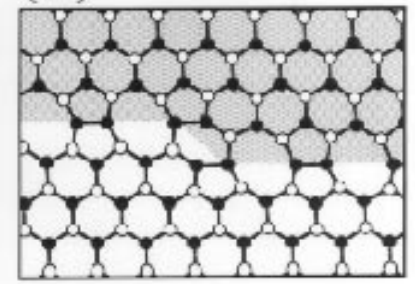

Figure 6. Core structure of kinks in the $30^{\circ}$ partial, and associated transition state. Kink notation is explained in the text. (a) LK kink. (b) LK' kink. (c) RK kink. (d) RK' kink.

The energies for LK and LK' given in Table II, show that reconstruction produces low energy kinks in this case, as compared to the energy of the (unreconstructed) soliton. At first sight, the formation energy for these reconstructed defects is expected to be mostly associated with the local strain at the kink cores. The Keating-model results can give us a qualitative understanding of these local-strain effects. The LK is found to add little additional strain to that imposed by the core reconstruction itself, with a slightly negative formation energy, while for the LK' kink we compute a Keating formation energy of $0.44 \mathrm{eV}$. Note that the trend in energies for the two left kinks is in qualitative agreement with the TB results. This is actually true for all four kink types, as can be seen by looking at the kink energies in the fourth column Table II. For the LK saddle-point configuration we computed an energy barrier of $1.52 \mathrm{eV}$. This result is in very good agreement with experimental estimates. Such a high barrier to motion can be understood by the presence of severe bond-bending and stretching distortions at the core of the defect, along with the presence of malcoordinated atoms. Bond angles as small as $50.4^{\circ}$ are found, as well as bonds stretching to $2.80 \AA$.

Also shown in Table II are the formation energies for RK $(1.24 \mathrm{eV})$ and RK' $(1.85 \mathrm{eV})$. These values are surprisingly high, since both are being fully reconstructed. No single structural feature of the right kinks could be traced in order to explain the unexpected formation energies. The minimum and maximum distortions of bond lengths and angles do not vary drastically among the four kink types. In Ref. [4], the strain fields and the corresponding energies associated with these kink are analyzed in more detail (where we find the trends in energy among the various kinks to be determined by the medium-range strain introduced by the defect, in 
each case). Like in the case of the left kinks, the rather high migration barrier of $2.03 \mathrm{eV}$ for the right kinks is associated with the drastic bond distortions and malcoordination of atoms at the core. Note that this barrier is substantially higher than the 1.52 value we obtained for the left kinks, leading to a physical picture of "fast" and "slow" plasticity carriers for the $30^{\circ}$ partial dislocation.

Kinks and solitons can be considered as the fundamental types of excitations in the dislocation cores. Important structural features and modes of dislocation dynamics can also be associated with the complexes formed by these basic defect types. Here, we consider the energetics of the kink-soliton complexes. The important questions concern whether or not these complexes form bound states, as well as the associated binding energies and migration barriers. We considered each of the kink-soliton complexes in two configurations. The left complex ( $\mathrm{LC}=\mathrm{LK}+$ soliton) was studied in the state of closest approach, LC(0), in which the two constituents overlap and cannot be distinguished; and also in an extended state, LC(1), in which the soliton and the kink have been separated to adjacent positions. We also analyzed the corresponding right-complex cases, $\mathrm{RC}(0)$ and $\mathrm{RC}(1)$. The results are shown in Table II. We find that the soliton binds strongly with both the left and the right kinks, in agreement with Ref. [6]. However, contrary to what is found in that work, our results indicate the LC to be more strongly bound than the RC. From the binding energies and the energies of these more extended configurations, we obtain a lower bound of $0.80 \mathrm{eV}(\mathrm{LC})$ and 0.49 (RC) for the dissociation barrier of these bound states. It should be emphasized that these results are in sharp contrast with those for kink-soliton complexes in the SP reconstruction of the $90^{\circ}$ partial dislocation, which are found to be unstable. Finally, we note that the energy of the LC is lower than that of the soliton, making the former the more likely site for unpaired electrons in the core of the $30^{\circ}$ partial.

\section{Comparison with experi- mental results}

The results for the formation energies and migration barriers of kinks in the $90^{\circ}$ and $30^{\circ}$ partial dislocations are summarized in Table III. For the $30^{\circ}$ partial, of the two equilibrium states of each kink [(LK,LK') and $\left.\left(\mathrm{RK}, \mathrm{RK}^{\prime}\right)\right]$, one is to be regarded as an intermediate metastable state in the propagation of the kink, in view of the substantial difference in formation energy between the two states. The state with the lower formation energy will determine the kink concentration in each case (this being the number included in Table III). For comparison, we include the ranges of experimental results for both quantities, obtained from different techniques.[18-21] We observe that, for the $30^{\circ}$ partial, our values are in excellent agreement with the experimental ones.

One possible interpretation of these experiments is offered by theory of Hirth and Lothe, in which the dislocation velocity is given by $v_{d} \propto 2 \times$ $\exp \left[-\left(U_{k}+W_{m}\right) / k T\right]$, where $U_{k}$ is the kink formation energy and $W_{m}$ is the kink migration barrier. This equation is written assuming the equivalence between the two kinks resulting from the nucleation of a stable double kink (a kink-antikink pair). This assumption does not hold for the $30^{\circ}$ partial, where the left and right kinks are intrinsically different. A more general form, given by

$$
v_{d} \propto \exp \left[-\frac{1}{2 k T}\left(U_{L K}+U_{R K}\right)\right] \times\left[\exp \left(-\frac{W_{m}^{L K}}{k T}\right)+\exp \left(-\frac{W_{m}^{R K}}{k T}\right)\right]
$$

must be used. The quantity of interest in the first activated term is the average formation energy of the two kink species. The second term is derived from the kink velocities, and therefore the relative velocity appears in the generalized form. In the $30^{\circ}$ partial this term is dominated by the velocity of the left kinks (fast carriers), given the much higher migration barrier of the right kinks (slow carriers). We stress that the average formation energy of the kink-antikink pairs in Table III falls within the range of the experimental numbers, for the $30^{\circ}$ partial. An alternative theory of dislocation glide has been proposed, $[28,29]$ in which the motion is controlled by the pinning of kinks by strong obstacles along the dislocation line, and the kink migration barriers are not rate controlling. Our work does not address such pinning mechanisms, thus being unable to decide between the latter and the HL theories. Nevertheless, our results are certainly consistent with the HL interpretation.

Finally, despite the fact that we did not consider the true ground state for the $90^{\circ}$ partial (the excellent agreement we obtain for the kink barriers being somewhat fortuitous) our results are qualitatively consistent with the experimental images in Ref. [21], which show 
a higher concentration of kinks in the $90^{\circ}$ partial. In Table III, we see that kink energies are lower in this dislocation, as compared to the $30^{\circ}$ partial. Obviously, this is only plausible to the extent that this general trend of lower kink energies carries over to the groundstate DP structure of the $90^{\circ}$ partial.

\section{Conclusion}

The main results of our study of the atomic structure of the $30^{\circ}$ and the $90^{\circ}$ partial dislocations in homopolar semiconductors, as reported in Refs. [2-5], can be summarized as follows. In $\mathrm{Si}, \mathrm{C}$, and Ge, the DP structure of the $90^{\circ}$ partial is found to be lower in energy than the SP geometry. The energy difference between the two structures follows the same trends as the stiffness and the size of the gap in these materials. In $\mathrm{Si}$, our investigation of solitons and kinks in the $30^{\circ}$ partial and the SP structure of the $90^{\circ}$ partial shows a picture of reconstructed kinks in both cases, with energies and migration barriers that compare favorably with experimental estimates. Solitons are shown to be very mobile in the $90^{\circ}$ partial, and less so in the $30^{\circ}$ partial. Kink-soliton complexes are found to be unstable or marginally so in the $90^{\circ}$ partial, while in the case $30^{\circ}$ partial such complexes appear stable, with binding energies of at least $0.5 \mathrm{eV}$.

\section{References}

[1] V. V. Bulatov, J. F. Justo, W. Cai, and S. Yip, Phys. Rev. Lett. 79, 5042 (1997).

[2] R. W. Nunes, J. Bennetto, and D. Vanderbilt, Phys. Rev. Lett. 77, 15417 (1996).

[3] J. Bennetto, R. W. Nunes, and D. Vanderbilt, Phys. Rev. Lett. 79, 245 (1997).

[4] R. W. Nunes, J. Bennetto, and D. Vanderbilt, Phys. Rev. B 57, 10388 (1998).

[5] R. W. Nunes, J. Bennetto, and D. Vanderbilt, Phys. Rev. B 58, 12563 (1998).

[6] V. V. Bulatov, S. Yip, and A. S. Argon, Philos. Mag. A 72, 453 (1995).

[7] J. R. K. Bigger et al., Phys. Rev. Lett. 69, 2224 (1992); L. B. Hansen et al., Phys. Rev. Lett. 75, 4444 (1995); T. A. Arias and J. D. Joannopoulos, Phys. Rev. Lett. 73, 680 (1994).

[8] R. Jones, J. Phys. (Paris), Colloq. 40, C6-33 (1979); Philos. Mag. B 42, 213 (1980); R. Jones et al, Phys. Status Solidi 138, 369 (1993).

[9] S. Marklund, Phys. Status Solidi B 92, 83 (1979); J. Phys. (Paris), Colloq. 44, C4-25 (1983).

[10] J. R. Chelikowsky, J. C. H. Spence, Phys. Rev. B 30, 694 (1984).

[11] K. W. Lodge et al., Philos. Mag. A 60, 643 (1989).
[12] S. Marklund and Y.-L. Wang, Phys. Status Solidi B 82, 137 (1992); Solid State Commun. 91, 301 (1994).

[13] M. I. Heggie and R. Jones, Philos. Mag. B 48, 365 (1983); 48379 (1983); Inst. Phys. Conf. Ser. 87, 367 (1987) ; M. I. Heggie, R. Jones, and A. Umerski, Phys. Stat. Sol 138, 383 (1993).

[14] S. Öberg, P. K. Sitch, R. Jones, and M. I. Heggie, Phys. Rev. B 51, 13138 (1995).

[15] P. B. Hirsch, Mater. Sci. Tech. 1, 666 (1985).

[16] M. S. Duesbery and G. Y. Richardson, Crit. Rev. Solid State Mater. Sci. 17, 1 (1991).

[17] H. Alexander and H. Teichler, in Materials Science and Technology, edited by R. W. Cahn, P. Hassen, and E. J. Kramer (VCH Weiheim, Cambridge, 1993), Vol. 4, p. 249.

[18] H. Gottschalk et al., Phys. Stat. Sol. (a) 138, 547 (1993).

[19] B. Ya. Farber, Yu. L. Iunin, and V. I. Nikitenko, Phys. Stat. Sol. (a) 97, 469 (1986); B. Ya. Farber et al., ibid. 138, 557 (1993).

[20] V. I. Nikitenko, B. Ya. Farber, and Yu. L. Iunin, Sov. Phys. JETP 66, 738 (1987).

[21] H. R. Kolar, J. C. H. Spence, and H. Alexander, Phys. Rev. Lett. 77, 4031 (1996).

[22] X.-P. Li, R. W. Nunes, and D. Vanderbilt, Phys. Rev. B 47, 10891 (1993).

[23] In our calculations, the supercell sizes were chosen by converging the defect energies with respect to defectdefect interactions, starting from smaller cells. More detailed descriptions of the supercells can be found in Refs. [2, 3, 4, 5]

[24] N. Lehto and S. Öberg, Phys. Rev. Lett. 80, 5568 (1998).

[25] R. W. Nunes and D. Vanderbilt, unpublished.

[26] P. N. Keating, Phys. Rev. 145, 637 (1966).

[27] G.-X. Qian and D. J. Chadi, J. Vac. Sci. Technol. B 4, $1079(86)$.

[28] V. Celli, M. Kabler, T. Ninomiya, and R. Thomson, Phys. Rev. 131, 58 (1963).

[29] V. Rubin and A. Orlov, Sov. Phys. Solid State 11, 2635 (1970). 
Table I. Calculated energy difference in $\mathrm{meV} / \AA$, between the SP- and DP-core reconstructions of the $90^{\circ}$ partial in $\mathrm{C}, \mathrm{Si}$, and Ge. Cell size refers to the double-period cell. $E_{\mathrm{DP}}$ is the energy of the double-period reconstruction. For the single-period case, $\bar{E}_{\mathrm{SP}}$ and $\Delta E_{\mathrm{SP}}$ are respectively the average and difference of the energies for the two different relative arrangements of mirror symmetry-breaking.

\begin{tabular}{lrrrr}
\hline & \multicolumn{2}{c}{192 -atom supercell } & \multicolumn{3}{c}{588 -atom supercell } \\
& $E_{\mathrm{DP}}-\bar{E}_{\mathrm{SP}}$ & $\Delta E_{\mathrm{SP}}$ & $E_{\mathrm{DP}}-\bar{E}_{\mathrm{SP}}$ & $\Delta E_{\mathrm{SP}}$ \\
\hline $\mathrm{C}$ LDA & & & & \\
$\quad$ & -235 & 126 & - & - \\
TETB & -100 & 74 & -76 & 14 \\
Keating & -21 & 123 & 34 & 24 \\
$\quad$ Keating [1] & -121 & 160 & - & - \\
$\mathrm{Si}$ & & & & \\
LDA & -69 & 48 & - & - \\
TETB & -75 & 39 & -57 & 3 \\
Keating & -27 & 40 & -7 & 8 \\
Keating [1] & -40 & 67 & - & - \\
Ge & & & & - \\
LDA & -58 & 27 & -5 & 6 \\
Keating & -21 & 32 & - & - \\
Keating [1] & -12 & 36 & & -
\end{tabular}

[1] Evaluated at LDA-relaxed structure.

Table II. Formation energy of defects in the $30^{\circ}$ partial dislocation, in eV. Defect energies are referred to a defectfree dislocation core. Defect migration barrier is shown in the last column, for the pure kinks (LK's and RK's) and the soliton (S). For the defect complexes, last column gives the defect binding energy. Two different states are considered for each complex (the notation is explained in the text).

\begin{tabular}{lrr} 
& Formation energy & Migration barrier \\
\hline $\mathrm{S}$ & 1.33 & 0.30 \\
$\mathrm{LK}$ & 0.35 & 1.53 \\
$\mathrm{LK}$ & 0.76 & - \\
$\mathrm{RK}$ & 1.24 & 2.10 \\
$\mathrm{RK}{ }^{\prime}$ & 1.85 & - \\
\hline & & Binding energy \\
\hline $\mathrm{LC}(0)$ & 0.88 & 0.80 \\
$\mathrm{LC}(1)$ & 1.58 & - \\
$\mathrm{RC}(0)$ & 2.15 & 0.42 \\
$\mathrm{RC}(1)$ & 2.64 & - \\
\hline
\end{tabular}

Table III. Formation energy and migration barriers of dislocation kinks in Si, in eV. Range of available experimental estimates of is included.

\begin{tabular}{lrrr} 
Dislocation & Kink type & Formation energy & Migration barrier \\
\hline $30^{\circ}$ & LK & 0.35 & 1.53 \\
$30^{\circ}$ & RK & 1.24 & 2.10 \\
$90^{\circ}$ & LR & 0.12 & 1.62 \\
$90^{\circ}$ & RL & 0.12 & 1.62 \\
\hline Experiments & & $0.4-0.7$ & $1.2-1.8$ \\
\hline
\end{tabular}

